\title{
Reverse chemistry of iron under high pressure and the distribution of elements in the deep Earth
}

\section{Xiaoli Wang}

Linyi University https://orcid.org/0000-0003-3608-7163

\section{Xiaolei Feng}

University of Cambridge https://orcid.org/0000-0003-4410-4576

Jianfu Li

School of opto-electronic information science and technology, Yantai University

\section{Dalar Khodagholian}

California State University, Northridge

Jiani Lin

Linyi University

Matthew Jackson

UC Santa Barbara https://orcid.org/0000-0002-4557-6578

\section{Frank Spera}

University of California, Santa Barbara

\section{Simon Redfern}

Nanyang Technological University

Maosheng Miao ( $\nabla$ mmiao@csun.edu )

California State University, Northridge https://orcid.org/0000-0001-9486-1204

\section{Article}

Keywords: Earth, mantle, core, iron

Posted Date: December 31st, 2020

DOl: https://doi.org/10.21203/rs.3.rs-130002/v1

License: (c) (i) This work is licensed under a Creative Commons Attribution 4.0 International License.

Read Full License 


\title{
Reverse chemistry of iron under high pressure and the distribution of elements in the deep Earth
}

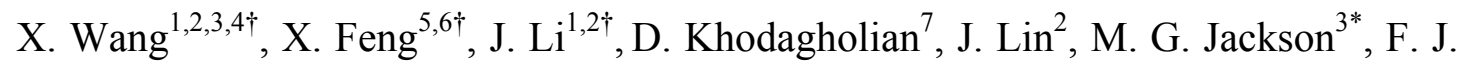
Spera $^{3}$, S. A. T. Redfern ${ }^{8,5, *} \&$ M. Miao ${ }^{7,4^{*}}$

${ }^{1}$ School of opto-electronic information science and technology, Yantai University, Yantai 264005, People's Republic of China

${ }^{2}$ School of Physics and Electronic Engineering, Linyi University, Linyi 276005, People's Republic of China.

${ }^{3}$ Department of Earth Science, University of California Santa Barbara, Santa Barbara, California 93110, United States.

${ }^{4}$ Beijing Computational Science Research Center, Beijing 100084, People's Republic of China.

${ }^{5}$ Center for High Pressure Science and Technology Advanced Research (HPSTAR), Shanghai 201203, China.

${ }^{6}$ Department of Earth Sciences, University of Cambridge, Downing Street, Cambridge, CB2 3EQ, UK.

${ }^{7}$ Department of Chemistry and Biochemistry, California State University, Northridge, California.

${ }^{8}$ Asian School of the Environment, Nanyang Technological University, 50 Nanyang Avenue, 639798, Singapore.

${ }^{\dagger}$ These authors contributed equally to this work.

${ }^{*}$ Correspondence and requests for materials should be addressed to: M.M. (email:mmiao@csun.edu), S.A.T.R. (email: simon.redfern@ntu.edu.sg) or to F.J.S. (email: jackson@geol.ucsb.edu)

\begin{abstract}
We demonstrate a remarkable change of chemical trend of iron under high pressure that is of great importance for understanding the distribution of elements in the Earth's mantle and core. Using a massive-scale first principles study, we show that while reacting with $p$-block elements under increasing pressure from ambient to that of Erath's core, iron tends to reverse its chemical nature, changing from an electron donor (reductant) to an electron acceptor, and oxidizes $p$-block elements. Such reverse chemistry significantly impacts the stoichiometries, bond types and strengths, structures and properties of iron compounds under deep planetary conditions, making
\end{abstract}


many $p$-block elements that are conventionally labeled lithophile or chalcophile to highly siderophile. The chemical binding strengths with iron show an inverse correlation with the depletion of $p$-block elements in silicate Earth. Furthermore, silicon shows a distinct anomaly in its bonding to iron, which suggests silicon may readily be incorporated into Earth's core. 
The distribution and abundance of both major and trace elements in the Earth's interior provide a record of its formation and evolution ${ }^{1,2}$. An understanding of this record demands knowledge of the chemical affinity of the elements and their compounds under the high-pressure conditions of Earth's interior. For many years, our understanding of such affinities has been predominantly biased by low-pressure observations that are of dubious applicability to Earth's deep mantle and core ${ }^{3}$. Many trace elements are found to have greatly reduced concentrations on Earth relative to their solar abundance ${ }^{4,5}$. This is usually explained in terms of either the escape of elements to space due to volatility during the high-energy conditions of terrestrial accretion $^{3}$, or the incorporation into the Earth's core ${ }^{6}$. The core sequestration model relies on the reactivity of trace elements with $\mathrm{Fe}$ (and $\mathrm{Ni}$ ) under high pressure, which is problematic to assess due to the difficulty of experimentally achieving terrestrial core pressures $(135-367 \mathrm{GPa})$.

Thanks to improvements in computational power and methods, the high-pressure chemistry of $\mathrm{Fe}$ has become accessible, leading to the discovery of a number of new Fe compounds with trace elements that supports the argument that they are incorporated in the core. For example, recent work showed that iron may actually bind strongly with xenon to form an $\mathrm{Fe}_{3} \mathrm{Xe}$ compound at the pressures of Earth's core, suggesting that core sequestration is the cause of the "missing xenon paradox ${ }^{9,8}$. A similar mechanism was suggested for the depletion of iodine in Earth ${ }^{9}$, although the volatility of the iodine renders this explanation ambiguous. The reactions of Fe with major elements such as $\mathrm{O}$ also become quite unusual at very high pressure. As revealed by both computer simulation and diamond anvil cell (DAC) experiments, iron can form an oxygen-rich $\mathrm{FeO}_{2}$ compound at the pressures of Earth's lower mantle, even if it remains in the low oxidation state of $+2^{10-12}$. We show here that these striking phenomena are all related to dramatic changes in 'iron chemistry' under high-pressure. The broad-ranging chemical trends of iron can only be revealed by a large-scale study of iron reactivity across the periodic table, a task that cannot be performed experimentally with reasonable resources and time.

Many recent studies show that first principles structure predictions are 
sufficiently advanced that enthalpies of compound formation at high pressure can be accurately calculated and the nature of the chemical bond elucidated ${ }^{7-9,12-17}$. Using this approach, we have systematically explored the bonding of iron with $p$-block elements in the periodic table. High pressure greatly enhances bonding to iron for many $p$-block elements that are conventionally labeled as lithophile or chalcophile ${ }^{1,18}$, making them highly siderophile. However, the depletion of the $p$-block elements in silicate Earth correlates inversely with Fe binding strength. This striking result suggests that although the Earth's core can host large quantities of the $p$ elements, it is not the cause of their depletion. Instead, cosmochemical accretion models that call on elemental loss by volatility during high-energy conditions of terrestrial accretion may be more relevant ${ }^{19}$. Furthermore, silicon shows a distinct anomaly in its bonding to iron, such that it becomes one of the strongest under high pressure, which suggests silicon may readily be incorporated into Earth's core, corroborating recent perspectives on the composition of Earth's core based on sound speed measurements, experimental petrology and seismology $\mathrm{y}^{20,21}$.

\section{Results and discussions}

We conducted massive-scale first-principles simulations studying the reactivity of Fe with most of the $p$-block elements and its dependence with increasing pressure. For each element $(\mathrm{X})$, the structures of a series of compositions $\left(\mathrm{Fe}_{\mathrm{n}} \mathrm{X}_{\mathrm{m}}\right)$ are searched by Particle Swarm Optimization (PSO) algorithm and density functional calculations. The selection of elements includes three major element $\mathrm{S}(721 \mathrm{~K} ; 50 \%$ condensation temperature of $664 \mathrm{~K})$, Si $(2628 \mathrm{~K} ; 1310 \mathrm{~K})$ and $\mathrm{P}(556 \mathrm{~K} ; 1229 \mathrm{~K})$ and a suite of geochemical tracers Ge (3103 K; 883 K), As (886 K; 1065 K), Se (958 K; 697 K), Sn (2543 K; $704 \mathrm{~K}), \mathrm{Sb}(2023 \mathrm{~K} ; 979 \mathrm{~K})$, and Te (1263 K; $709 \mathrm{~K})$ which are critical for understanding planetary accretion and core formation. (The temperatures in parentheses give the 1-bar boiling point and the 50\% equilibrium condensation temperature at $10^{-4}$ bar total pressure for solar system abundances of each element, respectively, as a guide to their volatility and reactivity in the condensing solar nebula.) Condensation temperatures are useful to consider when assessing if a 
particular element is deficient in the silicate mantle relative to chondrites because it suffered volatilization during planetary formation; it is a major task to explore whether such elemental deficiencies relate to elements being "hidden" in the metallic core.

The stability and its relation to element abundance in Earth interior Our calculations reveal that pressure can dramatically increase the stability of iron compounds formed with most $p$-block elements as evidenced by a significant decrease of formation enthalpy (Fig. 1). At ambient conditions, although some $2 p$ elements (e.g. $\mathrm{C}, \mathrm{N}, \mathrm{O})$ can form stable compounds with iron with a $\Delta \mathrm{H}_{\mathrm{f}}$ of approximately -0.5 eV/atom, a subset of $3 p$ (e.g. Si, P, S; Fe - Al compounds are excepted, and will be discussed separately) and $4 p$ (e.g. Ge, As, Se) elements bind only loosely with iron. This general trend of reactivity is markedly changed upon increasing pressure: most of these elements can form stable compounds with iron with a $\Delta \mathrm{H}_{\mathrm{f}}$ of at least $-1 \mathrm{eV} /$ atom. For example, $\mathrm{Fe}$ and Te are not likely to form a stable compound (with $\Delta \mathrm{H}_{\mathrm{f}}=-0.2 \mathrm{eV} /$ atom) at $0 \mathrm{GPa}$. However, $\Delta \mathrm{H}_{\mathrm{f}}$ decreases by $1 \mathrm{eV} /$ atom under high pressure, making the compound FeTe as stable as FeS at $300 \mathrm{GPa}$, a pressure similar to that in Earth's inner core of $367 \mathrm{GPa}$ ).

This pressure-enhanced Fe reactivity may promote the incorporation of many $p$-block elements, especially the heavier ones that were previously disregarded due to their weak or absent binding with Fe, into Earth's core. Like the previous works, our results first appear to support the model of core sequestration, i.e. the depletion of certain elements in the silicate Earth is due to their incorporation into Earth's core. However, the integrated picture that compares the abundance of elements and their binding strength with Fe across the $p$-block of the periodic table shows the opposite trend. The $p$-block element abundances, normalized to CI chondrites, are inversely correlated with their binding strength to Fe as quantified by the formation enthalpies of the most stable compounds (Figs. $2 \mathrm{a}-2 \mathrm{c}$ ), i.e. the stronger they bind with Fe the less they are depleted in the silicate Earth. While pressure increases, the correlation represented by the shaded stripes becomes steeper due to stronger binding to Fe (more negative enthalpy of formation) for the heavier elements such as $\mathrm{Se}, \mathrm{Te}, \mathrm{Bi}, \mathrm{Pb}$ etc. 
This inverse correlation clearly shows that reaction with Fe in Earth's core is unlikely to be the cause of the depletion of trace $p$-block elements in the silicate Earth, although such reactions may become exceedingly strong under terrestrial core conditions. One reason for the inverse correlations is that the volatility of an element (quantified by its condensation temperature) correlates inversely with its binding strength with $\mathrm{Fe}$, i.e. the higher the volatility the weaker the binding with $\mathrm{Fe}$ (Supplementary Fig. 1). Another factor is that binding of an element with Fe may prevent its evaporation in primordial Earth. Furthermore, our results are in accordance with the fact that moderately siderophile elements ( $\mathrm{Ni}, \mathrm{Co}, \mathrm{W}$ etc.) are depleted in silicate Earth whereas the highly siderophile elements (Re, Os, Ir, Ru etc.) are not ${ }^{22}$.

The reversal of Fe chemistry under pressure. A topological investigation of the electron density is conducted, employing Bader charge analysis ${ }^{23,24}$. The charge transfer between iron and $p$-block elements shows that, apart from $\mathrm{Al}$ and $\mathrm{Si}$ compounds (see below), the magnitude of charge transfer changes almost linearly with atomic number in each period from group 13 to group 17 (Figs. $2 \mathrm{~d}-2 \mathrm{f}$ ). At 0 $\mathrm{GPa}$, the charge is negative for most of the $p$-block elements, indicating that iron is oxidized. The charge transfer is generally small for heavy $p$-block elements, which explains the poor stability of their Fe compounds. Generally, the charge transfer from iron to $p$-block elements decreases with increasing atomic number in the same group. For example, the Bader charges on $\mathrm{Fe}$ in pnictides increase in the order $\mathrm{N}>\mathrm{P}>\mathrm{As}>\mathrm{Sb}$. Similar increases can be found across the chalcogens and halogens. This order of increasing charge remains under increasing pressure (Figs. 2d-2f).

Under high pressure, the electrons redistribute toward Fe, reducing its positive charge. For heavy $p$-block elements, like Ge, P, As, Te and I, the charge on Fe changes from positive to negative at $0,150,110,30$, and $100 \mathrm{GPa}$, respectively (Figs. $2 \mathrm{~d}-2 \mathrm{f}$ ). This charge transfer reversal (CTR) changes the chemical character of iron from being a reductant (electron donor) at ambient pressure to an oxidant (electron acceptor) at Earth's core conditions. For example, when combining with iodine, the charge on iron changes from positive to negative at $100 \mathrm{GPa}$. In other word, iron iodide becomes iodine ferride above $100 \mathrm{GPa}$. Similar CTR also happens in $\mathrm{Fe}-\mathrm{B}$ 
and $\mathrm{Fe}$ - Se compounds, but at higher pressures of 375 and $405 \mathrm{GPa}$, respectively.

The charge redistribution mainly happens between $\mathrm{Fe} 3 d$ and $\mathrm{X} n p$ orbitals (Supplementary Fig. 2), which is the natural result of the energy shifts of the $3 d$ and the $n p$ bands (Supplementary Fig. 3). The Fe $3 d$ bands become lower in energy because they have a smaller radius and are therefore less prone to change under increasing pressure (Supplementary Fig. 4).

Structural evolution of iron compounds. Structure evolution of Fe compounds is also affected by charge redistribution as pressure increases. At low pressure, many structures contain lone pair electrons on $p$-block elements that will disappear under high pressure, accompanying by the increase of coordination number and charge transfer back to Fe (Figs. 3a - 3d for Fe-I; Figs. 3e - 3g for Fe-As and Fe-Te). Both of the two stable Fe-I compounds adopt layered structures at ambient pressure, including $\mathrm{FeI}_{2}$ in $\mathrm{MoS}_{2}(P \overline{3} m 1)$ and $\mathrm{FeI}_{3}$ in $\mathrm{FeBr}_{3}(R \overline{3})$ structure with lone pairs on iodine anions pointing toward the space between layers (Figs. 3a and 3b). However, at 150 and $300 \mathrm{GPa}$, iron-rich compounds adopt densely-packed structures, including $\mathrm{Fe}_{2} \mathrm{I}$ adopting $\mathrm{Ni}_{2} \mathrm{In}$ structure $\left(P 6_{3} / m m c\right)$ (Fig. 3c) and $\mathrm{Fe}_{2} \mathrm{I}$ adopting $\mathrm{Cu}_{3} \mathrm{Au}$ structure $(F m \overline{3} m)$ (Fig 3d). Correspondingly, the coordination number of iodine increases substantially, to 6 in $\mathrm{Fe}_{2} \mathrm{I}$ and 12 in $\mathrm{Fe}_{3} \mathrm{I}$, and the lone pairs disappear, in good accordance with the CTR under pressure. Similar structure evolution is also found in other compounds containing lone pair electrons at ambient pressure, including Fe-As and Fe-Te compounds. (See Fig. 3e for ambient phase of both $\mathrm{FeAs}_{2}$ and $\mathrm{FeTe}_{2}$, Figs. $3 \mathrm{f}$ and $3 \mathrm{~g}$ and for high-pressure phases of FeAs and FeTe, respectively.) In contrast, no lone pair is found in the low-pressure structures of $\mathrm{Fe} X$ where $X$ is a group 13 or 14 element. Furthermore, some compounds contain Fe - Fe bonds in the low-pressure structure that simply vanish as pressure increases (Fig. 3h). For example, FeSn and FeGe are stable in a highly symmetric $P 6 / \mathrm{mmm}$ structure that contains $\mathrm{Fe}-\mathrm{Fe}$ inter-metallic bonds (Fig 3h). While increasing pressure induces the large charge transfer to iron, $\mathrm{Fe}-\mathrm{Fe}$ bonds disappear and the compounds become more ionic. At last, it is remarkable that many $\mathrm{FeX}$ compounds adopt the simple $\mathrm{CsCl}$ structure under high pressure, due to the large charge transfer to Fe. At pressures above $150 \mathrm{GPa}$, 
FeSn, FeGe and FeSi transform into the $\mathrm{CsCl}$ structure $(P m \overline{3} m)$ (Fig 3i) which is a common structure for $\mathrm{AB}$ type ionic compounds when the radius of $\mathrm{A}^{+}$and $\mathrm{B}^{-}$ions are similar, as stated by Pauling's first rule. Complete structures and structural parameters are shown in Supplementary Fig. 5 and Supplementary Table 1. All the structures are dynamically stable. The phonon spectra of Fe-I compounds are shown in Supplementary Fig. 6.

The anomaly of Si. An distinctive feature emerging from the comprehensive computation of $\mathrm{Fe}$ chemistry is an anomaly in reacting with Si and Al. Unlike the general trend regarding the change of electron density described earlier, the charges of $\mathrm{Fe}$ at $0 \mathrm{GPa}$ increase in the order $\mathrm{C}>\mathrm{Ge}>\mathrm{Sn}>\mathrm{Si}$ for group 14 elements. The charges of Fe for the last three elements are very close to each other. Under high pressure, the charge of $\mathrm{Fe}$ in $\mathrm{Fe}-\mathrm{Si}$ compounds decreases most dramatically and becomes significantly lower than Ge and Sn. A similar phenomenon can be seen for group 13 elements.

This distinctive anomaly is due to the fact that the unoccupied $d$ shells are significantly higher in energy for $\mathrm{Si}$ (and $\mathrm{Al}$ ) and cannot host electrons, in contrast to heavier $p$-block elements, leaving large charge transfer from $\mathrm{Si}$ (and $\mathrm{Al}$ ) to $\mathrm{Fe}$, especially under high pressure. At $300 \mathrm{GPa}$, the charge on iron in $\mathrm{FeSi}$ is as low as $-2 e$, indicating the very strong ionic nature of the compound. Indeed, the ELF values between Fe and Si decrease dramatically under pressure (Fig. $4 \mathrm{a}-4 \mathrm{~b}$ ). Consequently, Fe-Si bonding persistently strengthens at increasing pressure. At $0 \mathrm{GPa}$, the $\mathrm{Fe}-\mathrm{Si}$ bond strength is similar to Fe-S and Fe-P, all much weaker than Fe-O (Fig. 4c). Under increasing pressure, the Fe-Si binding strengthens most significantly and even surpasses $\mathrm{Fe}-\mathrm{O}$ at $250 \mathrm{GPa}$, implying that $\mathrm{Si}$ becomes highly siderophile at the pressure of Earth's core ${ }^{25}$. The lower density of FeSi (Supplementary Fig. 7) under core pressures can also explain the low core density as revealed by the seismic wave measurements.

$\mathrm{Ca} / \mathrm{Al}$ and $\mathrm{Mg} / \mathrm{Si}$ ratios are expected to exist in chondritic relative proportions in the silicate Earth, but both ratios are higher than chondritic in the shallower, accessible Earth. This suggests that either volatility controlled fractionation of $\mathrm{Mg} / \mathrm{Si}$ 
or the presence of an $\mathrm{Al}$ or Si-rich domain in Earth's deep interior ${ }^{26-28}$. Si and Al enrichment in the Earth's core may explain the higher-than-chondritic ratios in the shallower, accessible Earth.

\section{Summary}

We conducted a massive-scale first principles study on the reactivity of Fe with most of the $p$-block elements under pressures ranging from ambient to that of the center of the Earth. For each element X, the stability of Fe-X compounds with various compositions under different pressures are studied after searching the most stable structures using PSO algorithm and DFT calculations. While piecing together the results of all the elements, we found a general and solid trend of Fe chemistry, namely, under increasing pressure, Fe tends to become more electron negative and may revert its chemical from a reductant to an oxidant while reacting with many $p$-block elements. This chemical trend of $\mathrm{Fe}$ might have profound effect to the element distribution in Earth interior because it renders that pressure can significantly enhance the binding strength of these elements with Fe. However, while comparing the Fe binding strengths with the depletion of the element in silicate Earth, we found a distinct inverse correlation, which strongly supports the volatile depletion mechanism. On the other hand, the revealed strong binding with Fe under core condition provides a strong chemical driving force for the incorporation of light $p$ elements in Earth's core. Among them, Si shows an anomaly of exceedingly strong binding with $\mathrm{Fe}$, suggesting it a key component that lowers the density of the Earth's core.

We would like to emphasize that this work does not aim to develop a comprehensive thermodynamic model of Earth's core for which many other important factors need to be included — but is instead focused on identifying new chemistries for Fe at terrestrial core conditions. For example, Earth's core may contain a considerable budget of light elements such as $\mathrm{S}, \mathrm{O}$ and $\mathrm{H}$ that might modify trace element affinities for the core ${ }^{21}$. However, since the Earth's core consists of predominantly Fe (and Ni) that will largely lower the activity of the light elements, the inclusion of these light elements is unlikely to overturn the chemistry of $p$-block elements in the core that has 
been examined here. Furthermore, our calculations are performed for crystalline compounds, and are therefore more directly related to Earth's solid inner core. On the other hand, the general trend that the chemical binding with Fe becomes much stronger under pressure can be equally applied to the liquid outer core, because the chemical driving force is irrelevant to the state of the matter. Furthermore, the change of the Fe redox propensity under increasing pressure can also help to understand and perceive the redox state of Earth mantle and its evolution during the accretion of the Earth and the segregation of the core. ${ }^{29}$

\section{Methods}

Structure searches under pressure. We performed structure predictions through a global minimization of free energy surfaces based on the CALYPSO (Crystal structure AnaLYsis by Particle Swarm Optimization) methodology as implemented in CALYPSO code $\mathrm{e}^{30,31}$. We searched the structures of stoichiometric $\mathrm{Fe}_{m} \mathrm{X}_{n}(m=1-3 ; \mathrm{n}=1-3)$ with simulation cell sizes of 1-4 formula units under pressures of $150 \mathrm{GPa}$ and $300 \mathrm{GPa}$, respectively. All the structures are optimised at a higher accuracy.

Structure searches under pressure. In addition, The calculations for local structural relaxations and electronic properties were performed in the framework of density functional theory within the generalized gradient approximation Perdew-Burke-Ernzerhof (GGA-PBE) $)^{32}$ and frozen-core all-electron projector-augmented wave $(\mathrm{PAW})$ method $^{33,34}$ as implemented in the VASP code ${ }^{35}$. A cutoff energy of $700 \mathrm{eV}$ and appropriate Monkhorst-Pack ${ }^{36} k$-mesh with $k$-points density $0.03 \AA^{-1}$ were chosen to ensure that all the enthalpy calculations were well converged to less than $1 \mathrm{meV} /$ atom.

Formation enthalpy calculations. For the most stable structures at each pressure, the formation enthalpy per atom is calculated using the following formula:

$$
H_{f}\left(\mathrm{Fe}_{m} \mathrm{X}_{n}\right)=\left[H\left(\mathrm{Fe}_{m} \mathrm{X}_{n}\right)-m H(\mathrm{Fe})-n H(\mathrm{X})\right] /(m+n)
$$

where $H_{f}$ is the formation enthalpy per atom and $H$ is the calculated enthalpy per chemical unit for each compound. The enthalpies for Fe and $X$ are obtained from the 
most stable structures as searched by the CALYPSO method at the desired pressures.

Data availability. All data that are necessary to reproduce the results are included in this published article (and its supplementary materials files). The VASP code can be acquainted at www.vasp.at. The CALYPSO code can be acquainted at www.calypso.cn.

\section{References}

1. McDonough, W. F. \& Sun, S. -s. The composition of the Earth. Chem. Geol. 120, 223-253 (1995).

2. Anderson, D. L. The inner inner core of Earth. Proc. Natl. Acad. Sci. 99, 13966-13968 (2002).

3. Wood, B. J., Walter, M. J. \& Wade, J. Accretion of the Earth and segregation of its core. Nature 441, 825 (2006).

4. Anders, E. \& Grevesse, N. Abundances of the elements: Meteoritic and solar. Geochim. Cosmochim. Acta 53, 197-214 (1989).

5. Wang, Z. \& Becker, H. Ratios of S, Se and Te in the silicate Earth require a volatile-rich late veneer. Nature 499, 328-331 (2013).

6. Lodders, K. \& Fegley, B. The planetary scientist's companion. (Oxford University Press on Demand, 1998).

7. Zhu, L., Liu, H., Pickard, C. J., Zou, G. \& Ma, Y. Reactions of xenon with iron and nickel are predicted in the Earth's inner core. Nat. Chem. (2014). doi:10.1038/nchem.1925

8. Stavrou, E. et al. Synthesis of Xenon and Iron-Nickel Intermetallic Compounds at Earth's Core Thermodynamic Conditions. Phys. Rev. Lett. 120, 96001 (2018).

9. Du, X. et al. Structures and Stability of Iron Halides at the Earth's Mantle and Core Pressures: Implications for the Missing Halogen Paradox. ACS Earth Sp. Chem. 2, 711-719 (2018).

10. Liu, J. et al. Hydrogen-bearing iron peroxide and the origin of 
ultralow-velocity zones. Nature 551, 494-497 (2017).

11. Liu, J. et al. Altered chemistry of oxygen and iron under deep Earth conditions. Nat. Commun. 10, 1-8 (2019).

12. Botana, J. \& Miao, M.-S. Pressure-stabilized lithium caesides with caesium anions beyond the -1 state. Nat. Commun. 5, 4861 (2014).

13. Feng, J., Hennig, R. G., Ashcroft, N. W. \& Hoffmann, R. Emergent reduction of electronic state dimensionality in dense ordered Li-Be alloys. Nature 451, 445 (2008).

14. Zhang, W. et al. Unexpected Stable Stoichiometries of Sodium Chlorides. Science (80-. ). 342, 1502 LP - 1505 (2013).

15. Miao, M. S. Caesium in high oxidation states and as a p-block element. Nat. Chem. (2013). doi:10.1038/nchem.1754

16. Oganov, A. R. \& Ono, S. Theoretical and experimental evidence for a post-perovskite phase of MgSiO3 in Earth D layer. Nature 430, 445 (2004).

17. $\mathrm{Hu}, \mathrm{Q}$. et al. $\mathrm{FeO} 2$ and $\mathrm{FeOOH}$ under deep lower-mantle conditions and Earth's oxygen-hydrogen cycles. Nature 534, 241 (2016).

18. Vernikovsky, V. A. \& Sobolev, N. V. The main ideas of NL Dobretsov developed by his students and teammates. Russ. Geol. Geophys. 57, 3-7 (2016).

19. Lodders, K. Solar system abundances and condensation temperatures of the elements. Astrophys. J. 591, 1220 (2003).

20. Goncharov, A. F. et al. Effect of composition, structure, and spin state on the thermal conductivity of the Earth's lower mantle. Phys. Earth Planet. Inter. 180, 148-153 (2010).

21. Badro, J., Côté, A. S. \& Brodholt, J. P. A seismologically consistent compositional model of Earth's core. Proc. Natl. Acad. Sci. 111, 7542-7545 (2014).

22. Rubie, D. C. et al. Highly siderophile elements were stripped from Earth's mantle by iron sulfide segregation. Science (80-. ). 353, 1141-1144 (2016).

23. Bader, R. F. W. Atoms in molecules. Acc. Chem. Res. 18, 9-15 (1985). 
24. Henkelman, G., Arnaldsson, A. \& Jónsson, H. A fast and robust algorithm for Bader decomposition of charge density. Comput. Mater. Sci. 36, 354-360 (2006).

25. Lin, J.-F., Heinz, D. L., Campbell, A. J., Devine, J. M. \& Shen, G. Iron-silicon alloy in Earth's core? Science (80-. ). 295, 313-315 (2002).

26. Walter, M. J., Nakamura, E., Trønnes, R. G. \& Frost, D. J. Experimental constraints on crystallization differentiation in a deep magma ocean. Geochim. Cosmochim. Acta 68, 4267-4284 (2004).

27. Rubie, D. C. et al. Heterogeneous accretion, composition and core-mantle differentiation of the Earth. Earth Planet. Sci. Lett. 301, 31-42 (2011).

28. Jackson, C. R. M., Ziegler, L. B., Zhang, H., Jackson, M. G. \& Stegman, D. R. A geochemical evaluation of potential magma ocean dynamics using a parameterized model for perovskite crystallization. Earth Planet. Sci. Lett. 392, 154-165 (2014).

29. Armstrong, K., Frost, D. J., McCammon, C. A., Rubie, D. C. \& Ballaran, T. B. Deep magma ocean formation set the oxidation state of Earth's mantle. Science (80-. ). 365, 903-906 (2019).

30. Wang, Y., Lv, J., Zhu, L. \& Ma, Y. Crystal structure prediction via particle-swarm optimization. Phys. Rev. B 82, 094116 (2010).

31. Wang, Y., Lv, J., Zhu, L. \& Ma, Y. CALYPSO: A method for crystal structure prediction. Comput. Phys. Commun. 183, 2063-2070 (2012).

32. Perdew, J. P., Burke, K. \& Ernzerhof, M. Generalized Gradient Approximation Made Simple. Phys. Rev. Lett. 77, 3865-3868 (1996).

33. Blöchl, P. E. P. Projector augmented-wave method. Phys. Rev. B 50, 1795317979 (1994).

34. Kresse, G. \& Joubert, D. From ultrasoft pseudopotentials to the projector augmented-wave method. Phys. Rev. B 59, 1758-1775 (1999).

35. Kresse, G. \& Furthmüller, J. Efficient iterative schemes for ab initio total-energy calculations using a plane-wave basis set. Phys. Rev. B 54, 11169$11186(1996)$. 
36. Monkhorst, H. J. \& Pack, J. D. Special points for Brillouin-zone integrations. Phys. Rev. B 13, 5188-5192 (1976).

37. Jain, A. et al. Commentary: The Materials Project: A materials genome approach to accelerating materials innovation. APL Mater. 1, 011002 (2013).

38. Silivi, B. \& Savin, A. Classification of chemical bonds based on topological analysis of electron locatlized functions. Nature 371, 683-686 (1994).

\section{Acknowledgements}

This work was funded by the National Science Foundation CAREER award (No. 1848141 to M.M.), the American Chemical Society Petroleum Research Fund (No. 59249-UNI6 to M.M.), the National Natural Science Foundation of China (No. 11674144 and No. 11974154 to X.W.) and the Natural Science Foundation of Shandong Province (No. JQ201602 and No. 2019GGX103023 to X.W., and No. ZR2018MA038 to J.L.), the China Scholarship Council (X.F.), and the UK Natural Environment Research Council (No. NE/P012167/1 to S.A.T.R.). We thank the NSF-funded XSEDE resources (TG-DMR130005), the Stampede cluster run by Texas Advanced Computing Center and National Supercomputer Center Tianjin (China) for the supporting of computational resources. M.M. thanks Haiqing Lin from Beijing Computational Science Research Center for discussion. X.F. and S.A.T.R. thank Helen Williams from department of Earth Sciences, university of Cambridge for discussion.

\section{Author contributions}

X.W., X.F. and J.L. made equal contributions, and carried out most of the calculations. M.M., M.G.J., F.J.S. and S.A.T.R. coordinated and guided the research. M.M. conceived the idea of reverse chemistry of Fe and designed the study. M.M., M.G.J., F.J.S., X.F. and S.A.T.R. perceived and consolidated the significance of the new Fe chemistry to Earth science topics including the missing elements in silicate Earth and the incorporation of silicon in Earth's core. X.W., X.F, J.L, D.K. and M.M. were involved in electronic structure and chemical bonding analysis; X.F.,S.A.T.R., 
F.J.S.,M.G.J and M.M. were involved in the data analysis and the discussion of the influence of the new Fe chemistry to Earth science. M.M., M.G.J, F.J.S, S.A.T.R, X.F., and X.W. wrote the manuscript.

\section{Additional information}

Supplementary Information accompanies this paper at https://doi.org/.............

Competing interests: The authors declare no competing interest.

\section{Figures and Tables}

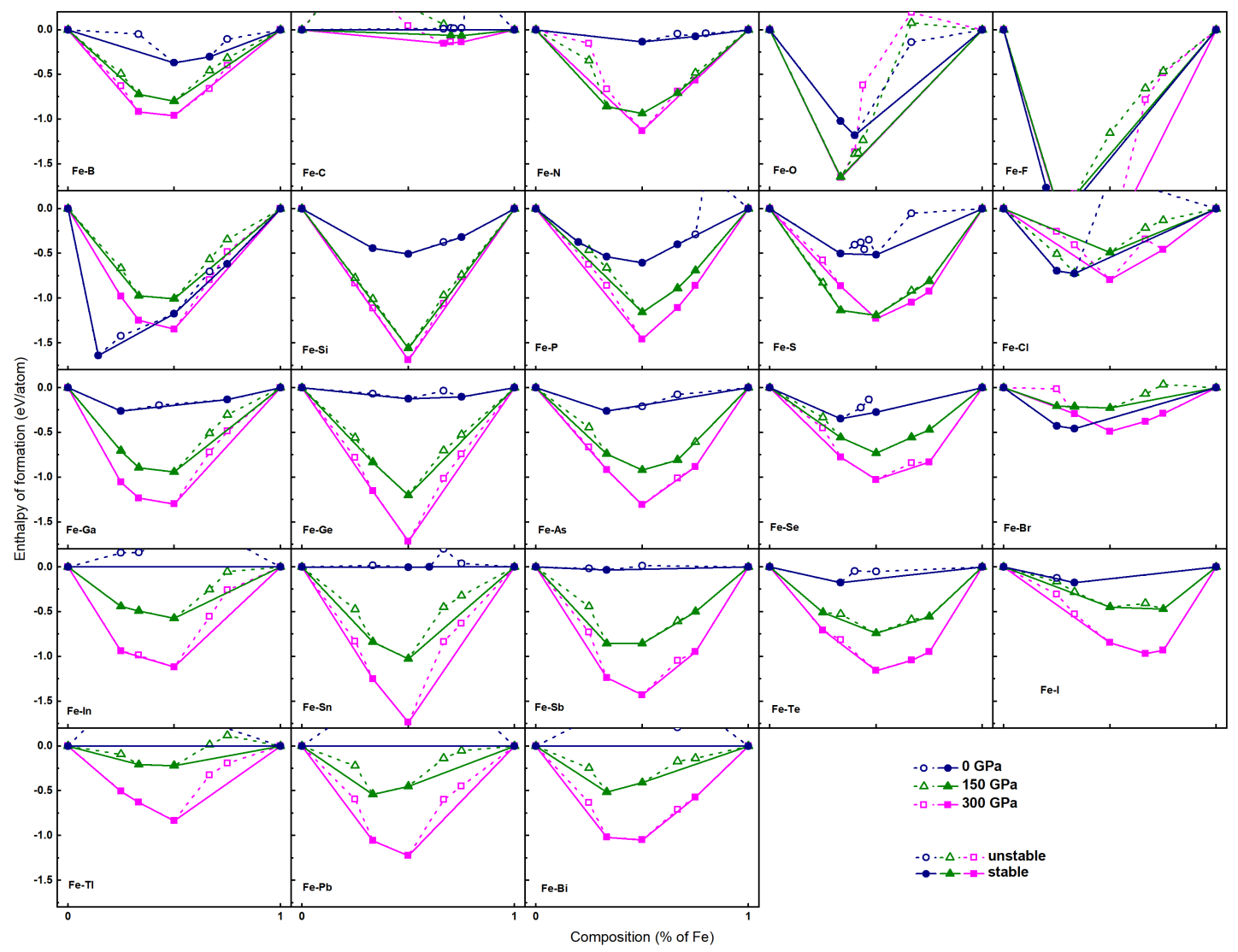

Fig. 1 Thermodynamic stability of main $p$-block - iron compounds at both ambient and high pressures. Most structures at ambient pressure are chosen from Materials Project ${ }^{37}$. Most structures of $\mathrm{Fe}_{\mathrm{m}} \mathrm{X}_{\mathrm{n}}(m / n=1-3)$ at high pressures are obtained from crystal structure searches using PSO algorithm ${ }^{30,31}$ (details are shown in Methods). Convex hulls are shown as solid lines, with stable compounds shown by solid symbols. Unstable compounds (open symbols) sit above convex hulls, with dotted lines indicating possible decomposition routes. 

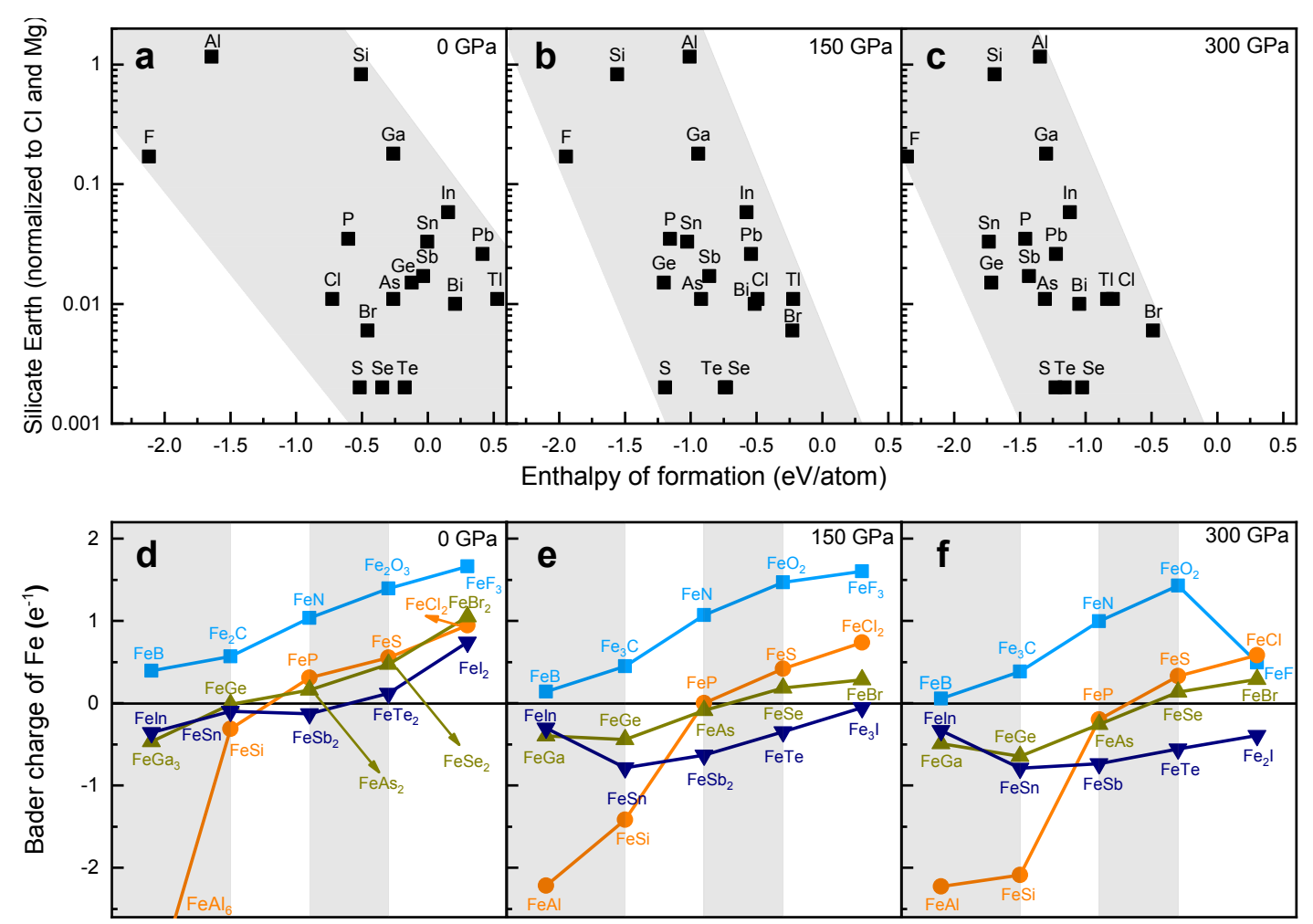

Fig. 2 Binding strength with Fe, their correlation with depletion in Earth and their chemical origin. a-c The correlations between element depletions and their binding strength with Fe under 0, 150 and $300 \mathrm{GPa}$. Horizontal axes show the enthalpy of formation per atom of the most stable Fe compound of selected element. The vertical axes show the abundance of an element normalized to CI chondrite and Mg. d-f Calculated Bader charge of iron in Fe - $X$ compounds at 0, 150 and $300 \mathrm{GPa}$. 
a.

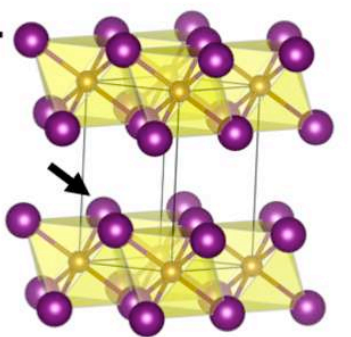

d.

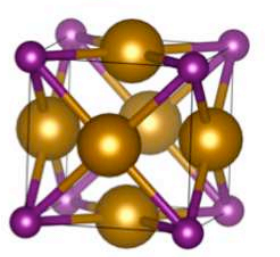

g.

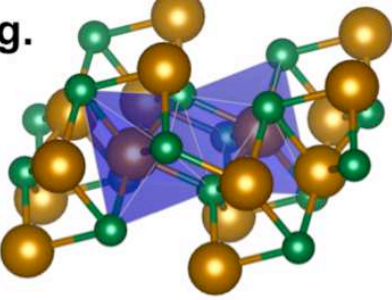

b.

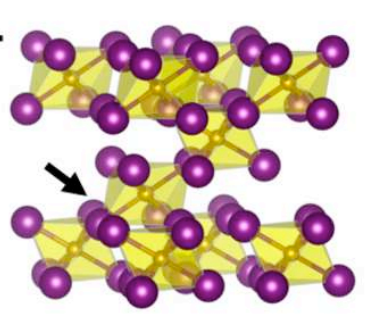

e.

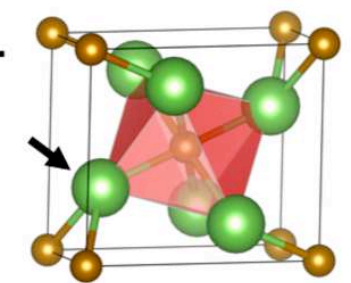

h.

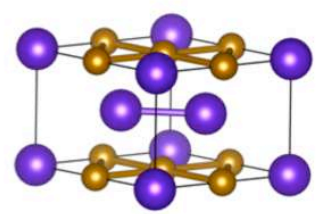

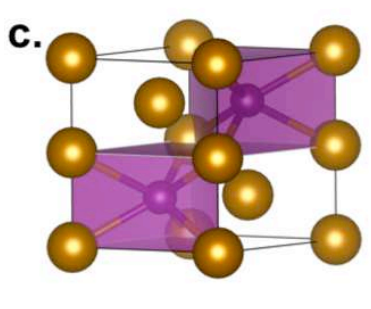

f.



i.

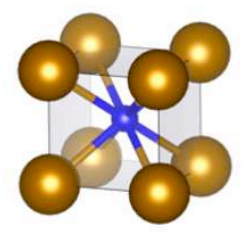

Fig. 3 Structure evolution of Fe-p block compounds under pressure. a layered structure of stable $\mathrm{FeI}_{2}$ at $0 \mathrm{GPa}$. b FeI 3 at $0 \mathrm{GPa}$; c Fe $\mathrm{Fe}_{2} \mathrm{I}$ at $150 \mathrm{GPa}$. d $\mathrm{Fe}_{3} \mathrm{I}$ at 150 GPa. e $\mathrm{Fe} X_{2}(X=\mathrm{As}$ and Te) structure at ambient pressure. f FeAs at $150 \mathrm{GPa}$. $\mathrm{g}$ FeTe at $150 \mathrm{GPa}$. $\mathbf{h} \mathrm{Fe} X(X=\mathrm{Sn}$ and $\mathrm{Ge})$ with intermetallic bonds between $\mathrm{Fe}$ atoms. i $\mathrm{CsCl}$ structure, a common structure for $\mathrm{Fe} X$ compounds under pressure. Brown balls represent $\mathrm{Fe}$ atoms; the size (small and large) indicates the charge transfer out of or into $\mathrm{Fe}$. The arrows show the interstitial space for lone pair electrons of $p$-block elements. 
a.

b.
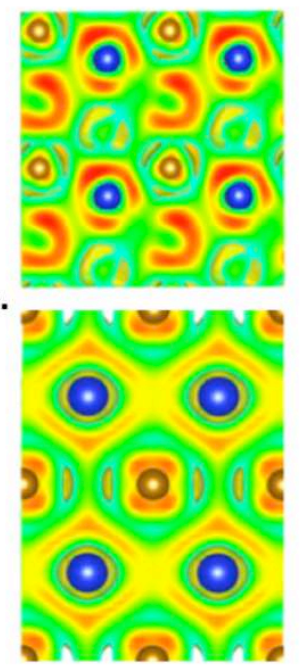

C.

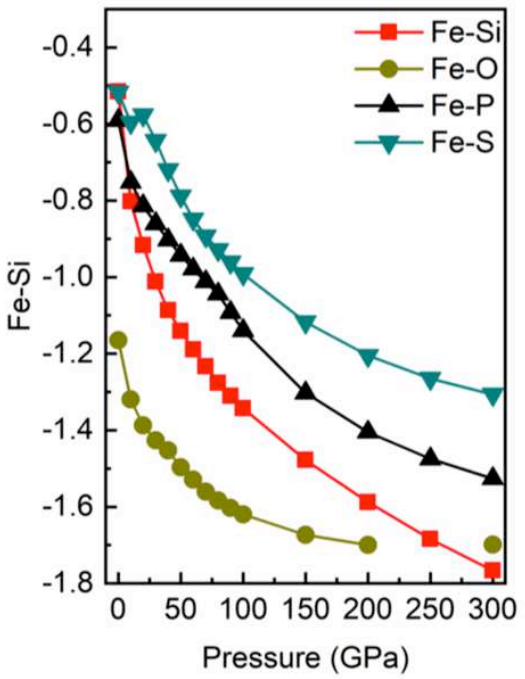

Fig. 4 Anomaly of Si in binding with Fe. Electron localization functions (ELF) ${ }^{38}$ of $\mathrm{FeSi} \mathbf{a}$ at $0 \mathrm{GPa}$ and $\mathbf{b}$ at $300 \mathrm{GPa}$. $\mathbf{c}$ the enthalpy of formation of Fe-X compounds as functions of pressure. The isosurfaces of the ELFs are taken at 0.2 . 


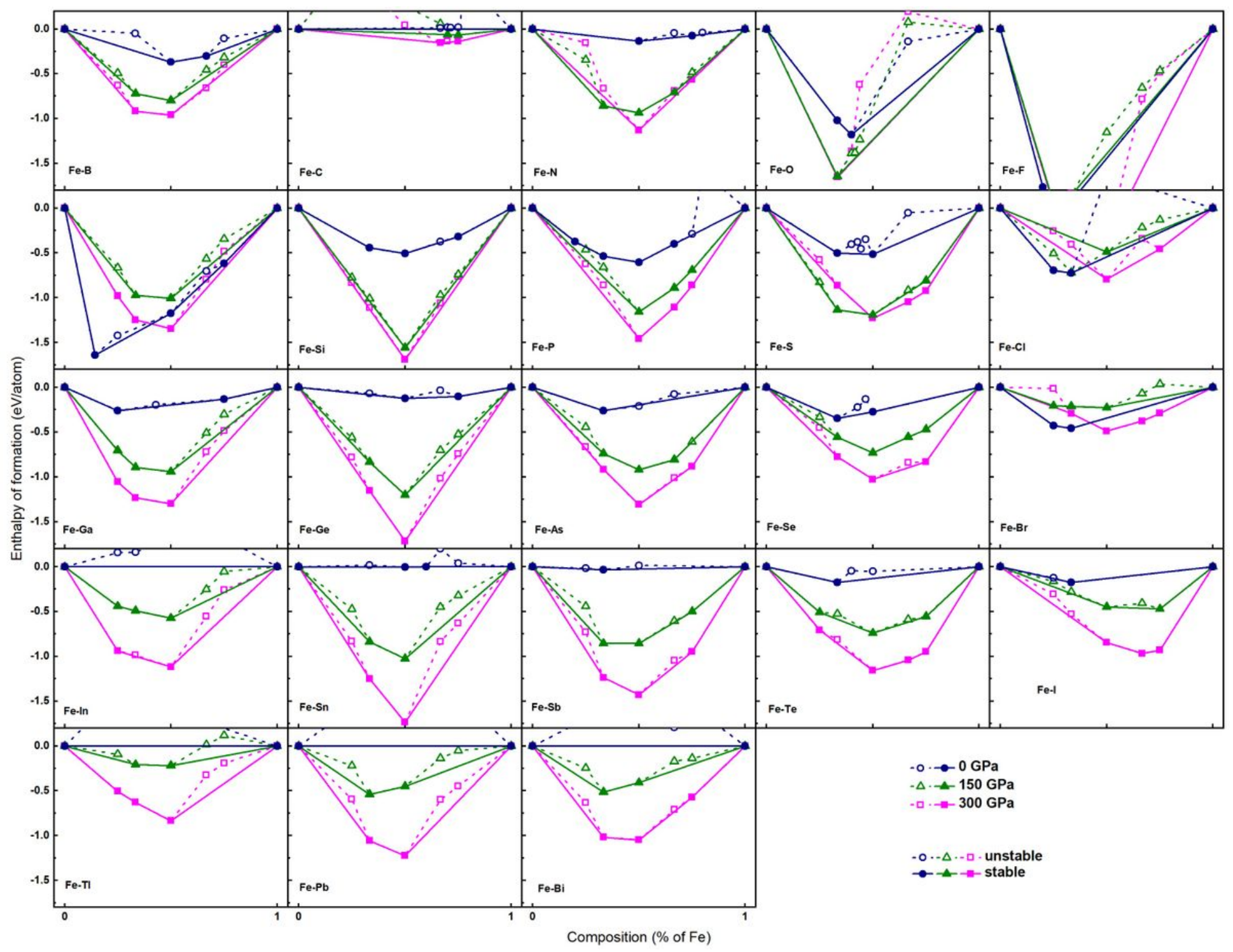

\section{Figure 1}

Thermodynamic stability of main p-block - iron compounds at both ambient and high pressures. Most structures at ambient pressure are chosen from Materials Project37. Most structures of FemXn $(m / n=1-$ 3) at high pressures are obtained from crystal structure searches using PSO algorithm 30,31 (details are shown in Methods). Convex hulls are shown as solid lines, with stable compounds shown by solid symbols. Unstable compounds (open symbols) sit above convex hulls, with dotted lines indicating possible decomposition routes. 



Figure 2

Binding strength with Fe, their correlation with depletion in Earth and their chemical origin. a-c The correlations between element depletions and their binding strength with Fe under 0, 150 and $300 \mathrm{GPa}$. Horizontal axes show the enthalpy of formation per atom of the most stable Fe compound of selected element. The vertical axes show the abundance of an element normalized to $\mathrm{Cl}$ chondrite and $\mathrm{Mg}$. $\mathrm{d}-\mathrm{f}$ Calculated Bader charge of iron in Fe - X compounds at 0, 150 and $300 \mathrm{GPa}$. 
a.

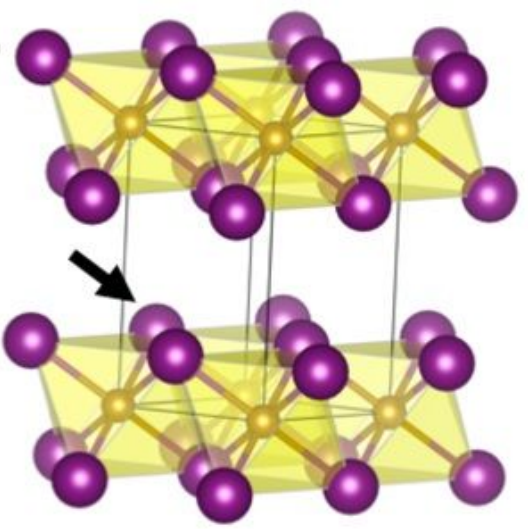

d.
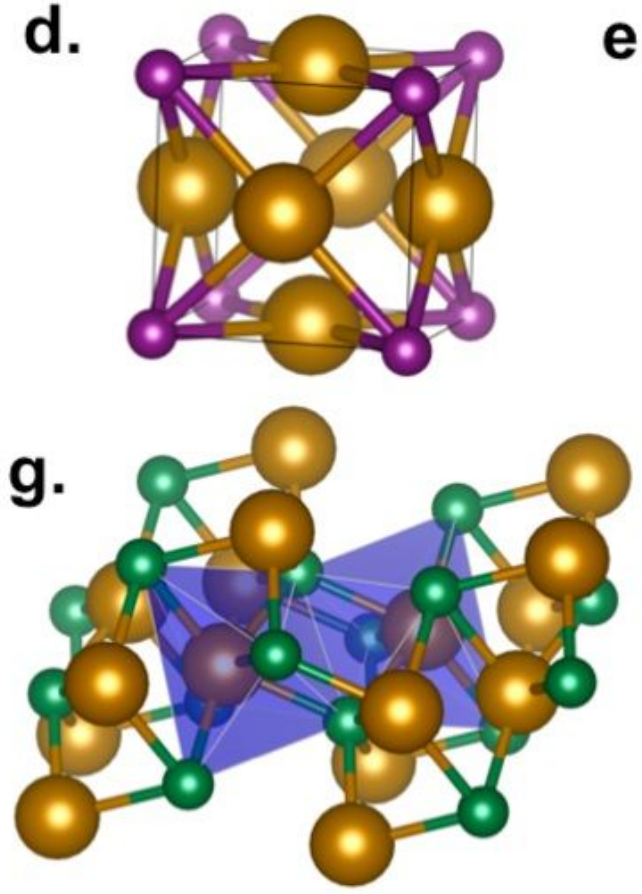

b.

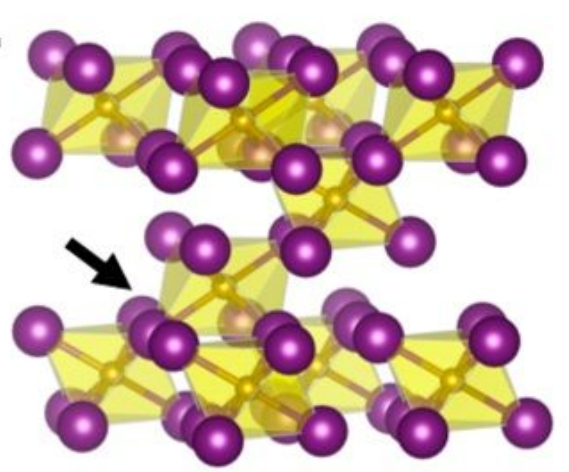

e.

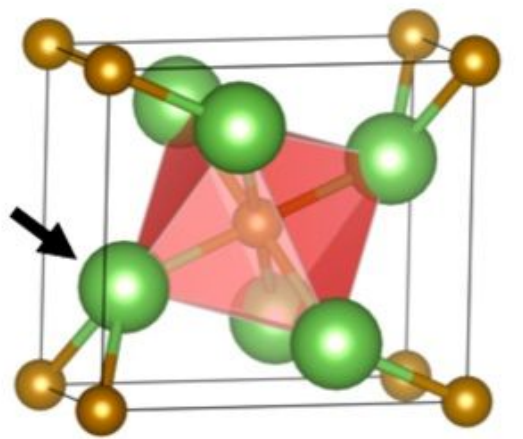

h.

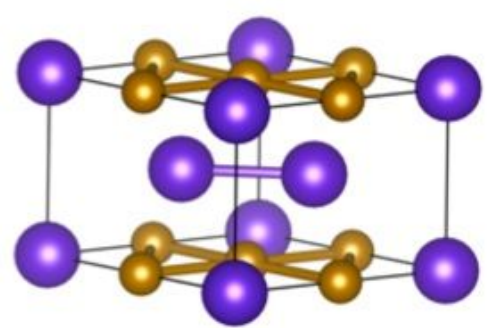

c.

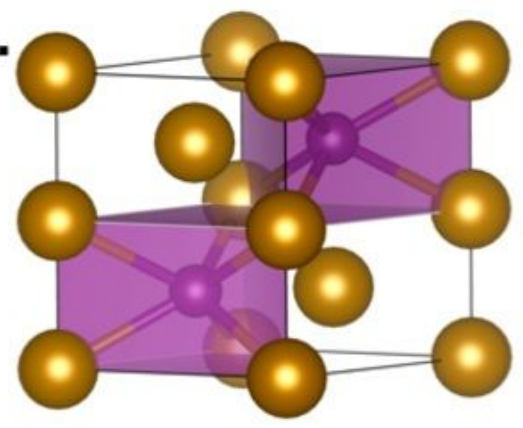

f.

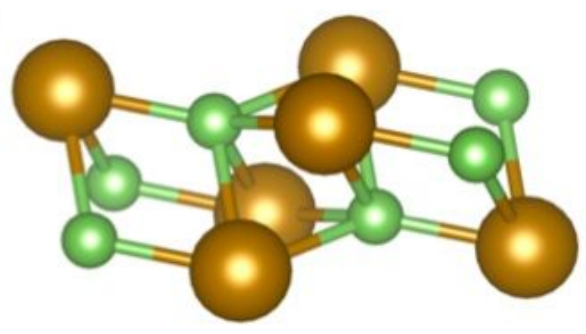

i.

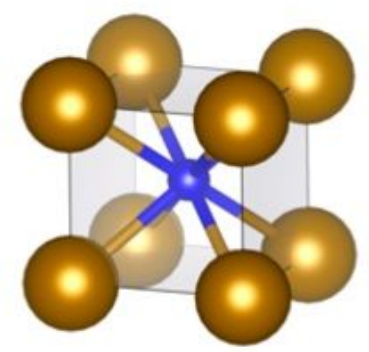

Figure 3

Structure evolution of Fe-p block compounds under pressure. a layered structure of stable Fel2 at $0 \mathrm{GPa}$. b Fel3 at $0 \mathrm{GPa}$; c Fe2I at $150 \mathrm{GPa}$. d Fe3I at $150 \mathrm{GPa}$. e FeX2 (X = As and Te) structure at ambient pressure. $f$ FeAs at $150 \mathrm{GPa}$. $g$ FeTe at $150 \mathrm{GPa}$. h FeX (X = Sn and $\mathrm{Ge}$ ) with intermetallic bonds between Fe atoms. i $\mathrm{CsCl}$ structure, a common structure for $\mathrm{FeX}$ compounds under pressure. Brown balls represent Fe atoms; the size (small and large) indicates the charge transfer out of or into Fe. The arrows show the interstitial space for lone pair electrons of p-block elements. 

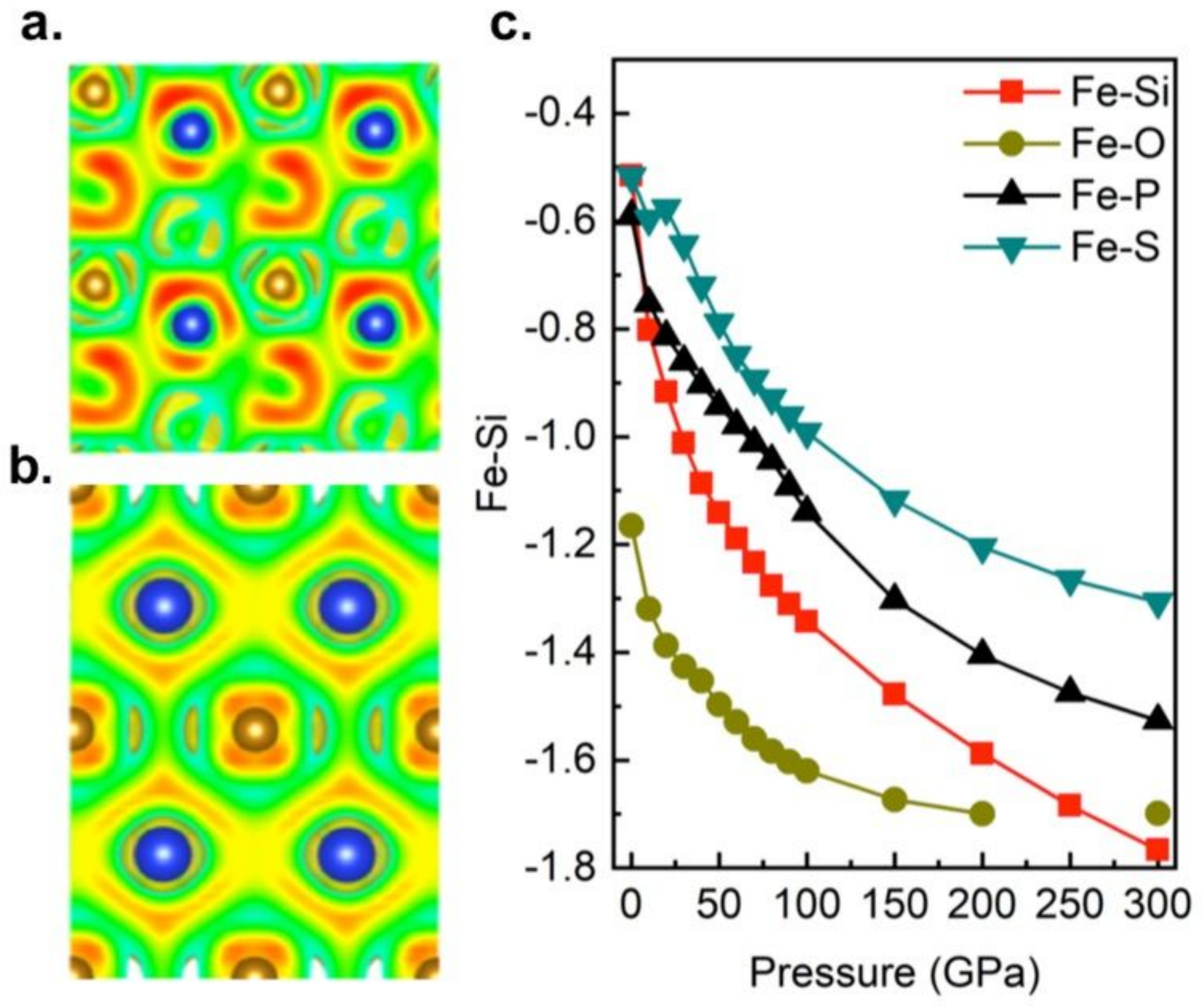

Figure 4

Anomaly of $\mathrm{Si}$ in binding with Fe. Electron localization functions (ELF) 38 of FeSi a at $0 \mathrm{GPa}$ and b at 300 $\mathrm{GPa}$. $\mathrm{c}$ the enthalpy of formation of Fe-X compounds as functions of pressure. The isosurfaces of the ELFs are taken at 0.2 .

\section{Supplementary Files}

This is a list of supplementary files associated with this preprint. Click to download.

- FeReverseChemistrySI.pdf 